\title{
On the Fourth-Order Accurate Compact ADI Scheme for Solving the Unsteady Nonlinear Coupled Burgers' Equations
}

\author{
Samir F. RADWAN \\ Department of Engineering Mathematics and Physics, Faculty of Engineering, \\ Alexandria University, Alexandria, Egypt \\ Received January 26, 1998; Accepted September 8, 1998
}

\begin{abstract}
The two-dimensional unsteady coupled Burgers' equations with moderate to severe gradients, are solved numerically using higher-order accurate finite difference schemes; namely the fourth-order accurate compact ADI scheme, and the fourth-order accurate Du Fort Frankel scheme. The question of numerical stability and convergence are presented. Comparisons are made between the present schemes in terms of accuracy and computational efficiency for solving problems with severe internal and boundary gradients. The present study shows that the fourth-order compact ADI scheme is stable and efficient.
\end{abstract}

\section{Introduction}

The basic flow equations describing unsteady transport problems form a parabolic hyperbolic system of partial differential equations. The interaction of the nonlinear convective terms and the dissipative viscous (or dispersion) terms in these equations can result in relatively severe gradients in the solution. Also, the accuracy of the numerical solution and the computational efficiency are highly dependent on the numerical methods used to solve this kind of partial differential equations. Standard three-point finite difference methods of approximating spatial derivatives may work well for smooth solutions, but they fail when severe gradients or discontinuities are present, which are common in the shock wave problems $[1,2,3]$. Lower-order accurate finite difference methods, such as upwindingtype finite differences, can be a remedy for the numerical oscillations and dispersions. However, they have a large amount of "numerical viscosity" that smooths the solution in much the same way that physical viscosity would, but to an extent that is unrealistic by several orders of magnitude, see Sharif and Busnaina [4]. Standard four-point finite difference methods, such as Leonard's method [5], are good in their higher-order accuracies and in reducing numerical smearing effects. But, they are plagued by their generation 
of spurious oscillations or overshoots in the neighborhood of discontinuities and lack accuracy, as reported by Liu et al. [6], and the present author [7]. TVD finite difference schemes $[8,9]$, guarantee oscillation-free solutions, but they are limited to second-order accuracy. Third-order accurate TVD schemes are reported by Gupta et al. [10], and by Liu et al. [6]. However, in their studies, nonlinearities were either not present or played a minor role. Therefore, the perfect numerical methods should possess both higher-order accuracy and sharp resolution of discontinuities without excessive smearing. Moreover, higher-order accurate numerical methods are attractive for problems with long computational time or with required higher accuracy solutions. As mentioned by Orszag [11], that at the cost of slight additional computational complexity, the fourth-order schemes achieve results in the 5\% accuracy range with approximately half the spatial resolution in each space direction compared with the second-order schemes (i.e. a factor 8 fewer grid points in three dimensions). But, the objection to the standard higher-order schemes comes from the additional nodes necessary to achieve the higher-order accuracy. This precludes the use of implicit methods since the obtained matrix is not of tridiagonal form, and it is necessary to use fictitious nodes for the boundary conditions. Also, they do not allow easily for non-uniform grids, unless at the expense of the order of accuracy. On the other hand, the compact schemes that treat the function and its necessary derivatives as unknowns at the grid nodes, like the Pade scheme [40], are fourth-order accurate, and compact in sence that they reduce to tridiagonal form. The compact schemes generally consist of finite difference schemes which involve two or three grid points. The three-point schemes fall into two classes. The first class consists of methods which are fourth-order accurate for uniform grids, such as Kreiss scheme [11, 12], the Mehrstellen method [13], the operator compact implicit scheme [14-16], and the Hermitian finite difference method of Peters [17]. The second class consists of methods that allow variable grids such as the cubic spline methods of Rubin and Graves [18-21], and the Hermitian finite difference method of Adam [22, 23]. For incompressible viscous flow problems, there has been several work on the construction of compact schemes for the incompressible Navier-Stokes equations, see for example, [24-33]. The most noted ones include the work of Gupta [24, 25]. He introduced a compact fourth-order finite difference scheme with three nodal points for the convection diffusion equations. His scheme does not seem to suffer excessively from spurious oscillatory behavior or numerical viscosity, and it converges with standard methods such as Gauss Seidel or SOR regardless of the diffusion [34, 35]. However, Yavneh [36], in his analysis of Gupta's scheme for the 2-D convection-diffusion equation with Dirichlet boundary conditions, has concluded that Gupta's compact scheme does not suffer from a cross-stream artificial viscosity, but it does include a streamwise artificial viscosity that is inversely proportional to the natural viscosity. For unsteady viscous flow cases, Weinan and Liu [37] introduced an modified version of Gupta's compact scheme based on the vorticity-stream function formulation. The requirement of compactness of this scheme is slighly relaxed (for the convection terms) so that the resulting scheme is simple, easy to implement, and with small phase error. They were successful to compute the driven cavity problem at high Reynold numbers upto $10^{6}$ on $1024^{2}$ grid, and they have concluded that the fourth-order compact methods are comparable in accuracy with the spectral methods for most problems of practical interests. For turbulent fluid flow, where there is a range of space and time scales, Lele [38] had introduced a series of higher-order compact schemes that are generalization of the Pade scheme with three nodal points, and with an improved 
representation of the shorter length scales. He applied them to the evolution of supersonic shear layers.

The disadvantage of the above higher-order compact schemes involving three nodal points is that the boundary conditions are no longer sufficient and they do not allow easily for non-uniform grids, unless at the expense of the order of accuracy. Another disadvantage of some compact schemes is the complexity of the resulting nonlinear finite difference equations and the associated difficulty in solving them efficiently. On the other hand, the compact scheme with two nodal points, like second-diagonal Pade scheme, is fourth-order accurate even for non-uniform spatial grids, and no fictitious points neither extra formula are needed for Dirichlet boundary conditions, as discussed by White [39] and Keller [40]. Also, Liniger and Willoughby [41] studied the numerical solutions of stiff systems of ordinary differential equations, that are encountered in many areas of applied mathematics, using compact two-point implicit methods. They introduced three main compact schemes with different order of accuracy, and with some very favorable properties. In particular, their schemes have $A$-stability in the sense of Dahlquist [55] and they account for the exponential character of the rapidly decaying solutions directly, which are referred as exponential fitting methods.

Inspite of many articles have appeared in the literature concerning the applications of the higher-order accurate schemes including the compact schemes to fluid dynamics problems, there is no much works done in the area of application of two-point compact schemes [39-44], like the fourth-order accurate second-diagonal Pade approximation, to multi-dimensional cases. This is the main objective of present study, where we study the feasibility of extending the two-point compact scheme to solve the unsteady twodimensional coupled Burgers' equations. They take the following form:

$$
\begin{aligned}
& \frac{\partial u}{\partial t}=-u \frac{\partial u}{\partial x}-v \frac{\partial u}{\partial y}+\nu \frac{\partial^{2} u}{\partial x^{2}}+\nu \frac{\partial^{2} u}{\partial y^{2}}, \\
& \frac{\partial v}{\partial t}=-u \frac{\partial v}{\partial x}-v \frac{\partial v}{\partial y}+\nu \frac{\partial^{2} v}{\partial x^{2}}+\nu \frac{\partial^{2} v}{\partial y^{2}}, \\
& x_{0} \leq x \leq x_{N}, \quad y_{0} \leq y \leq y_{M}, \quad t>0
\end{aligned}
$$

with the initial conditions

$$
u(x, y, 0)=u_{0}(x, y), \quad v(x, y, 0)=v_{0}(x, y)
$$

and the Dirichlet boundary conditions

$$
\begin{array}{ll}
u\left(x_{0}, y, t\right)=u_{1}(y), & u\left(x_{N}, y, t\right)=u_{2}(y), \\
u\left(x, y_{0}, t\right)=u_{3}(x), & u\left(x, y_{M}, t\right)=u_{4}(x), \\
v\left(x_{0}, y, t\right)=v_{1}(y), & v\left(x_{N}, y, t\right)=v_{2}(y), \\
v\left(x, y_{0}, t\right)=v_{3}(x), & v\left(x, y_{M}, t\right)=v_{4}(x),
\end{array}
$$

where $\nu$ is equal to $1 / R e>0$, and $R e$ is the Reynolds number. Coupled Burgers' equations are used to model many practical transport problems, such as vorticity transport, hydrodynamic turbulence, shock wave theory, wave processes in thermoelastic medium, transport 
and dispersion of pollutants in rivers, and sediment transport, see references [45-47]. The coupled Burgers' equations are an appropiate form of the Navier-Stokes equations. They have the same convective and diffusion form as the incompressible Navier-Stokes equations. Fletcher [48] made a comparison of finite element and finite difference methods with different orders of accuracy for solving the two-dimensional Burgers' coupled equations, and he concluded that the five-point finite difference scheme is the most efficient scheme. Boonkkamp and Verwer [49] have used the extrapolated Odd-Even Hopscotch scheme for solving the inhomogeneous two-dimensional coupled Burgers' equations, but their solution exhibited wiggles. Arminjon and Beauchamp [50] have concluded that the finite element method is efficient compared to the other methods, namely the method of lines and Runge-Kutta-type method in solving the above Burgers' equations. Jain and Raja [51] have used splitting-up technique to reduce the problem to a sequence of tridiagonal systems. Jain and Lohar [52] used spline locally one dimensional (SOLD) algorithm for solving coupled nonlinear parabolic equations. EL-Zoheiry and EL-Naggar [53] used the spline alternating direction implicit (SADI) method for solving the two-dimensional Burgers' equations. EL-Naggar [54] has presented a mixed implicit-explicit two levels algorithm that is based on SADI method for solving the coupled Burgers' equations. However, his results exhibited oscillations.

In the present study, higher-order accurate two-point compact alternating direction implicit algorithm (CADI) is introduced to solve the two-dimensional unsteady coupled Burgers' equations, for problems with moderate to severe internal and boundary gradients. The algorithm has the following features:

(1) it results in finite difference equations that involve only two-nodal points and therefore is formally fourth-order accurate on all grid points, even for non-uniform grids;

(2) it has $A$-stability in the sense of Dahlquist, and accounts for the exponential character of rapidly varying solutions [41];

(3) it utilizes Newton's method for linearization with a quardratic convergence;

(4) it requires only the given Dirichlet boundary conditions;

(5) the algorithm is simple and easy to implement.

In short, the present method is the natural extension of $A$-stable fourth-order accurate second-diagonal Pade approximation to solve multi-dimensional flow problems with moderate to severe gradients. Comparison of the present scheme with the fourth-order Du Fort Frankel scheme is made in terms of accuracy and computational efficiency, which show that the fourth-order compact ADI scheme is stable and efficient.

\section{The numerical schemes}

In this section, the present numerical schemes, namely the fourth-order accurate two-point compact ADI scheme and the fourth order Du Fort Frankel scheme, are derived for the two-dimensional unsteady coupled Burgers' equations (1). 


\subsection{Fourth-order accurate Du Fort Frankel scheme}

Let the interval $\left[x_{0}, x_{N}\right]$ be discretized into $N$ grid steps of size $\Delta x$, where $\Delta x=\left(x_{i}-x_{i-1}\right)$, $i$ is an index of any grid-point in $x$ direction. Similarly, the interval $\left[y_{0}, y_{M}\right]$ is discretized into $M$ grid steps of size $\Delta y$, where $\Delta y=\left(y_{j}-y_{j-1}\right), j$ is an index of any grid point in $y$-direction, and $n$ is an index for the temporal grid point. The explicit form of the Du Fort Frankel scheme for the two-dimensional coupled Burgers' equations (1), using Kreiss fourth-order accurate approximations [11] for the spatial derivatives, takes the following form:

$$
\begin{gathered}
{\left[\frac{\vec{\psi}_{i j}^{n+1}-\vec{\psi}_{i j}^{n-1}}{2 \Delta t}\right]=-u_{i j}^{n} D_{x}\left[1-\frac{\Delta x^{2}}{6} \delta_{x}^{2}\right] \vec{\psi}_{i j}^{n}+\nu \delta_{x}^{2}\left[1-\frac{\Delta x^{2}}{12} \delta_{x}^{2}\right] \vec{\psi}_{i j}^{n}} \\
-v_{i j}^{n} D_{y}\left[1-\frac{\Delta y^{2}}{6} \delta_{y}^{2}\right] \vec{\psi}_{i j}^{n}+\nu \delta_{y}^{2}\left[1-\frac{\Delta y^{2}}{12} \delta_{y}^{2}\right] \vec{\psi}_{i j}^{n},
\end{gathered}
$$

where

$$
\begin{aligned}
& \vec{\psi}=[u, v]^{T} \\
& D_{x} \vec{\psi}_{i j}=\frac{1}{2 \Delta x}\left(\vec{\psi}_{i+1 j}-\vec{\psi}_{i-1 j}\right) \\
& \delta_{x}^{2} \vec{\psi}_{i j}=\frac{1}{\Delta x^{2}}\left(\vec{\psi}_{i+1 j}-2 \vec{\psi}_{i j}+\vec{\psi}_{i-1 j}\right) .
\end{aligned}
$$

Define $c_{x}^{n}=u_{i j}^{n} \frac{\Delta t}{\Delta x}, c_{y}^{n}=v_{i j}^{n} \frac{\Delta t}{\Delta y}$, to be the local Courant numbers in $x$ and $y$ directions, $d_{x}=\nu \frac{\Delta t}{\Delta x^{2}}$, and $d_{y}=\nu \frac{\Delta t}{\Delta y^{2}}$. The above equation represents the fourth-order accurate leap-frog scheme for equations (1), and in order to obtain the final form of the fourthorder accurate explicit Du Fort Frankel scheme for the 2-D unsteady coupled Burgers' equations (1), the center node value $\left(\psi_{i j}\right)$ in the diffusion terms in equation (2.1) are replaced by their average at time-levels $(n-1)$ and $(n+1)$, giving:

$$
\begin{aligned}
& \vec{\psi}_{i j}^{n+1}=A \vec{\psi}_{i j}^{n-1}+B \vec{\psi}_{i+2 j}^{n}+C \vec{\psi}_{i+1 j}^{n}+D \vec{\psi}_{i-1 j}^{n}+E \vec{\psi}_{i-2 j}^{n} \\
& \quad+F \vec{\psi}_{i j+2}^{n}+G \vec{\psi}_{i j+1}^{n}+H \vec{\psi}_{i j-1}^{n}+L \vec{\psi}_{i j-2}^{n},
\end{aligned}
$$

where

$$
\begin{array}{ll}
A=\left(1-2.5 d_{x}-2.5 d_{y}\right) / Q, & B=\left(c_{x}-d_{x}\right) / 6 Q, \\
C=\left(-8 c_{x}^{n}+16 d_{x}\right) / 6 Q, & D=\left(8 c_{x}^{n}+16 d_{x}\right) / 6 Q, \\
E=-\left(c_{x}^{n}+d_{x}\right) / 6 Q, & F=\left(c_{y}^{n}-d_{y}\right) / 6 Q, \\
G=\left(-8 c_{y}^{n}+16 d_{y}\right) / 6 Q, & H=\left(8 c_{y}^{n}+16 d_{y}\right) / 6 Q, \\
L=-\left(c_{y}^{n}+d_{y}\right) / 6 Q, & Q=\left(1+2.5 d_{x}+2.5 d_{y}\right) .
\end{array}
$$




\subsection{Fourth-order accurate compact ADI scheme}

Liniger et al. [41] have introduced the following linear one step formulas for $\phi(x)$ containing real free parameters $(a \& b)$ :

$$
\begin{aligned}
& {\left[\phi_{i+1}-\phi_{i}\right]-\frac{\Delta x}{2}\left[(1+a) \phi_{x_{i+1}}+(1-a) \phi_{x_{i}}\right]+\frac{\Delta x^{2}}{4}\left[(b+a) \phi_{x x_{i+1}}-(b-a) \phi_{x x_{i}}\right]=e_{T}} \\
& e_{T}=\frac{\Delta x^{3}}{4} \int_{0}^{1}\left[2 \xi^{2}-2(1-a) \xi+(b-a)\right] \frac{\partial^{3}}{\partial x^{3}} \phi(x+\xi \Delta x) d \xi
\end{aligned}
$$

For the case of $a \geq 0$ and $b=1 / 3$, the resulting formula has a third-order accuracy. Moreover, for the case of $a=0$ and $b=1 / 3$, the formula has a fourth-order accuracy, which is known as the two-point second-diagonal Pade approximation:

$$
\begin{aligned}
& {\left[\phi_{i+1}-\phi_{i}\right]-\frac{\Delta x}{2}\left[\phi_{x_{i+1}}+\phi_{x_{i}}\right]+\frac{\Delta x^{2}}{12}\left[\Phi_{x x_{i+1}}-\phi_{x x_{i}}\right]=e_{T},} \\
& e_{T}=\frac{\Delta x^{5}}{24} \int_{0}^{1} \xi^{2}(\xi-1)^{2} \frac{\partial^{5}}{\partial x^{5}} \phi(x+\xi \Delta x) d \xi
\end{aligned}
$$

where $\phi_{x}, \phi_{x x}$ are the first and the second derivatives of the function $\phi(x)$. Using the above scheme and an ADI-type time marching procedure for the temporal derivative, the compact alternating direction implicit algorithm (CADI) for the coupled Burgers' equations (1), are obtained by first rewriting these equations as follows:

$$
\left[\begin{array}{l}
u \\
v
\end{array}\right]_{t}=\left[\begin{array}{c}
\left(\nu u_{x}-0.5 u^{2}\right)_{x} \\
\left(\nu v_{x}-u v\right)_{x}+v u_{x}
\end{array}\right]+\left[\begin{array}{c}
\left(\nu u_{y}-u v\right)_{y}+u v_{y} \\
\left(\nu v_{y}-0.5 v^{2}\right)_{y}
\end{array}\right],
$$

then the ADI - type time marching procedure requires, in one full time step, the solution of:

$$
\begin{gathered}
x \text {-sweep } \\
{\left[\begin{array}{l}
u_{t} \\
v_{t}
\end{array}\right]^{n+0.5}=\left[\begin{array}{c}
\left(\nu u_{x}-0.5 u^{2}\right)_{x} \\
\left(\nu v_{x}-u v\right)_{x}+v u_{x}
\end{array}\right]^{n+0.5}+\left[\begin{array}{l}
g_{1} \\
g_{2}
\end{array}\right]^{n},} \\
{\left[\begin{array}{l}
u_{x} \\
v_{x}
\end{array}\right]^{n+0.5}=\left[\begin{array}{l}
F \\
G
\end{array}\right]^{n+0.5},} \\
y \text {-sweep } \\
{\left[\begin{array}{l}
u_{t} \\
v_{t}
\end{array}\right]^{n+1}=\left[\begin{array}{c}
\left(\nu u_{y}-u v\right)_{y}+u v_{y} \\
\left(\nu v_{y}-0.5 v^{2}\right)_{y}
\end{array}\right]^{n+1}+\left[\begin{array}{l}
f_{1} \\
f_{2}
\end{array}\right]^{n+0.5},} \\
{\left[\begin{array}{l}
u_{y} \\
v_{y}
\end{array}\right]^{n+1}=\left[\begin{array}{l}
H \\
T
\end{array}\right]^{n+1},}
\end{gathered}
$$

where, $\left[f_{1}, f_{2}\right]^{T},\left[g_{1}, g_{2}\right]^{T}$ are the components of the first and the second matrices in the right hand side of equation (2.11). The solution procedure consists of solving, first, equations (2.12) in the solution vector $[U, V, F, G]^{T}$ at time level $n+0.5,(x$-sweep), then solving 
equations (2.13) in the solution vector $[U, V, H, T]^{T}$ at time level $n+1$, (y-sweep). Noting that $\alpha=1 / \Delta t$, and in order to apply the compact scheme to the solution in the $x$-sweep, a vector $\vec{Q}$ and its derivatives with respect to $x$, for Burgers equations (2.12), have been defined as follows:

$$
\begin{aligned}
& \vec{Q}_{i j}^{n+0.5}=\left[\begin{array}{c}
\nu u_{x}-0.5 u^{2} \\
\nu v_{x}-u v \\
\nu u \\
\nu v
\end{array}\right]_{i j}^{n+0.5}=\left[\begin{array}{c}
\nu F-0.5 U^{2} \\
\nu G-U V \\
\nu U \\
\nu V
\end{array}\right]_{i j}^{n+0.5} \\
& \vec{Q}_{x i j}^{n+0.5}=\left[\begin{array}{c}
u_{t} \\
v_{t}-v u_{x} \\
\nu F \\
\nu G
\end{array}\right]_{i j}^{n+0.5}-\left[\begin{array}{c}
g_{1} \\
g_{2} \\
0 \\
0
\end{array}\right]_{i j}^{n}=\left[\begin{array}{c}
\alpha U \\
\alpha V-V F \\
\nu F \\
\nu G
\end{array}\right]_{i j}^{n+0.5}-\left[\begin{array}{c}
g_{1}+\alpha U \\
g_{2}+\alpha V \\
0 \\
0
\end{array}\right]_{i j}^{n} \\
& \vec{Q}_{x x i j}^{n+0.5}=\left[\begin{array}{c}
F_{t} \\
G_{t}-v F_{x}-F v_{x} \\
u_{t}+u u_{x} \\
v_{t}+u v_{x}
\end{array}\right]_{i j}^{n+0.5}-\left[\begin{array}{c}
g_{1_{x}} \\
g_{2_{x}} \\
g_{1} \\
g_{2}
\end{array}\right]_{i j}^{n} \\
& =\left[\begin{array}{c}
\alpha F \\
\alpha G-V F_{x}-F G \\
\alpha U+U F \\
\alpha V+U G
\end{array}\right]_{i j}^{n+0.5}-\left[\begin{array}{c}
\alpha F+g_{1_{x}} \\
\alpha G+g_{2_{x}} \\
\alpha U+g_{1} \\
\alpha V+g_{2}
\end{array}\right]_{i j},
\end{aligned}
$$

where $g_{1}, g_{2}, g_{1 x}, g_{2 x}$ and $F_{x}$ in equations (2.15)-(2.16) are approximated by fourth-order accurate finite differences. Having substituted the vector $\vec{Q}$ and its derivatives into the above two-point second-diagonal Pade approximation, equation (2.9), by replacing $\phi_{i}$ by the vector $\vec{Q}$, we have four non-linear coupled finite difference equations in the solution vector $[U, V, F, G]^{T}$. Newton's method is used to linearize the equations, and the numerical solution is obtained by iteration. The resulting linearized equations form a block tridiagonal matrix system of order $N$, as in the following form:

$$
a_{i} \vec{\delta}_{i-1}+b_{i} \vec{\delta}_{i}+c_{i} \vec{\delta}_{i+1}=\vec{r}_{i}, \quad i=1,2, \ldots, N,
$$

where $a_{i}, b_{i}$, and $c_{i}$ are block matrices of order four, $\vec{\delta}=[\delta U, \delta V, \delta F, \delta G]^{T}$ is the change in the solution vector, and $\vec{r}$ is the right handside vector, each of order four. At each iteration, the LU-factorization algorithm is used to obtain the solution of the system (2.17). Similarly, the solution procedure of the Burgers' equation (2.11) in the $y$-sweep, using equations (2.13).

\subsection{Numerical stability limits}

The implicit formulation of the two-point compact scheme to the Burgers' equations is always unconditionally stable. In this case, the accuracy of the numerical solution depends on the size of the discretizations, and higher accuracy can be obtained by finer discretization. Moreover, the present higher-order scheme allows us to use large discretization in 
comparison with the second-order schemes. Also, it is well known that, for the convection diffusion equation, the leap-frog scheme is unconditionally unstable, while the Du Fort Frankel scheme has a stability limit $(c \leq 1 \& d>0)$ [56]. Therefore, it is necessary to use Von Neumann stability analysis to define the stability limit of the fourth-order accurate Du Fort Frankel scheme to Burgers' equations. In the present study, it will be sufficient to examine the stability of the fourth-order Du Fort Frankel scheme for one of the above coupled Burgers' equations (2.5), say for $u$-component. Let the numerical solution $U(x, y, t)$ be represented by a finite Fourier series, and for linear stability, we can examine the behaviour of a single term of the series, as follows:

$$
u(i \Delta x, j \Delta y, n \Delta t)=G(n \Delta t) e^{I\left[k_{x} i \Delta x+k_{y} j \Delta y\right]},
$$

where $G(t)$ is the amplitude function at time-level $\mathrm{n}$ of this term whose wave numbers in the $x$ and $y$ directions are $k_{x}$ and $k_{y}$, and $I=\sqrt{-1}$. Defining the $x$ and $y$ phase angles as $\theta_{x}=k_{x} \Delta x$ and $\theta_{y}=k_{y} \Delta y$, then, equation (2.18) becomes:

$$
u_{i j}^{n}=G^{n} e^{I\left[i \theta_{x}+j \theta_{y}\right]} .
$$

Substituting (2.19) into the first equation of (2.5), we obtain a quadratic equation for the amplification factor $\zeta$, its solution is:

$$
\zeta=\frac{G^{n+1}}{G^{n}}=\frac{1}{2}\left[\lambda \pm \sqrt{\lambda^{2}+4 A}\right]
$$

and

$$
\begin{aligned}
\lambda= & \frac{1}{3 Q}\left\{\left[\left(16 \cos \theta_{x}-\cos 2 \theta_{x}\right) d_{x}+\left(16 \cos \theta_{y}-\cos 2 \theta_{y}\right) d_{y}\right]\right. \\
& \left.+I\left[\left(-8 \sin \theta_{x}+\sin 2 \theta_{x}\right) c_{x}+\left(-8 \sin \theta_{y}+\sin 2 \theta_{y}\right) c_{y}\right]\right\},
\end{aligned}
$$

where $A$ and $Q$ are defined by equations (2.6). For the special case of $d_{x}=d_{y}=d$ and $c_{x}=c_{y}=c$, the modulus of the amplification factor $\zeta$, defined by the following equation:

$$
\chi\left(c, d, \theta_{x}, \theta_{y}\right)=\max \left(\left|\frac{1}{2}\left[\lambda+\sqrt{\lambda^{2}+4 A}\right]\right|,\left|\frac{1}{2}\left[\lambda-\sqrt{\lambda^{2}+4 A}\right]\right|\right)
$$

has been computed for different values of $|c|$ and $d$ and plotted, as shown in Fig. 1. This shows that the fourth-order accurate Du Fort Frankel scheme is unstable for the range $(0.35 \leq|c| \leq 1.0)$. E.g. $\chi(1,0.5, \pi / 2, \pi / 2)=1.77, \chi(1,0.5, \pi, \pi)=1.29, \chi(0.5,0.5, \pi / 2$, $\pi / 2)=1.14$, and $\chi(0.5,0.5, \pi, \pi)=1.29$. For small values of $|c|$ and $d(|c|<0.35)$, the instability only occurs for phase angles $\theta_{x}$ and $\theta_{y}$ close to $\pi$. Moreover, for smaller values of $d(d<0.1)$, the scheme has a neutral stability $(\chi=1.0)$. E.g. $\chi(0.25,0.5, \pi / 2, \pi / 2)=$ 0.87, $\chi(0.25,0.5, \pi, \pi)=1.29, \chi(0.25,0.01, \pi, \pi)=1.0$. Concerning the consistency of the present schemes, both of the schemes are consistent with the original differential equation (1). The finite difference equation using the compact ADI scheme is consistent in sense that the local truncation error, $e_{T}=O\left[\Delta x^{5}, \Delta t \Delta x^{2}, \Delta t \Delta x\right]$ tends to zero as $\Delta t$ and $\Delta x$ tend to zero. For Du Fort Frankel scheme equation (2.5) whose truncation error, $e_{T}=O\left[\Delta t^{2},(\Delta t / \Delta x)^{2}, \Delta x^{4}\right]$, the consistency condition requires the truncation error tends to zero upon $(\Delta t / \Delta x)^{2}$ approach zero as $\Delta t$ and $\Delta x$ approach zero. For this reason, 
and a much smaller time step than allowed by the above stability limit is implied. This concludes that each of the finite difference approximations to the 2-D coupled Burgers' equation, the fourth-order explicit Du Fort Frankel scheme and the compact scheme, satisfies the consistency condition. Then, the stability of the scheme will be the necessary and sufficient condition for convergence, which is true for linear PDE's. But, for the present nonlinear PDE's (1), the results of the test cases will verify the convergence, but with higher restricted stability limit.

\section{$3 \quad$ Numerical experiments}

For small value of $\nu$, Burgers' equation behaves merely as hyperbolic partial differential equation, and the problem becomes very difficult tosolve as steep shock-like wave fronts developed, as reported by Kreiss [57]. Therefore, the present higher-order schemes are applied to solve problems that are dominated by moderate to severe internal and boundary gradients.

\section{Problem case- -1}

The first test case is the solution of 2-D unsteady coupled Burgers' equations (1) in the domain $\{-1<x<1,0<y<\pi / 6 k\}$ with initial and Dirichlet boundary conditions given by the exact steady-state solutions, that are set to form moderate to severe internal and boundary gradients in the domain [48], as shown in Fig. 2a:

$$
u_{s}(x, y)=-\frac{2}{R e} \frac{\phi_{1}(x, y)}{\phi(x, y)}, \quad v_{s}(x, y)=-\frac{2}{R e} \frac{\phi_{2}(x, y)}{\phi(x, y)}
$$

where

$$
\begin{aligned}
& \phi(x, y)=a_{0}+a_{1} x+\left[e^{k(x-1)}+e^{-k(x-1)}\right] \cos k y, \\
& \phi_{1}(x, y)=a_{1}+k\left[e^{k(x-1)}-e^{-k(x-1)}\right] \cos k y, \\
& \phi_{2}(x, y)=-k\left[e^{k(x-1)}+e^{-k(x-1)}\right] \sin k y .
\end{aligned}
$$

The values of the parameters $\left(a_{0}, a_{1}, k, R e\right)$ determine the type of the gradient in the computed solutions. We consider three cases in this problem:

case 1a. moderate internal gradient with $a_{0}=a_{1}=110.13, k=5$ and $R e=10$;

case 1b. severe internal gradient with $a_{0}=a_{1}=1.2962 \times 10^{13}, k=25$ and $R e=50$;

case 1c. severe boundary gradient with $a_{0}=a_{1}=0.011013, k=5$ and $R e=10$.

The numerical steady-state solutions of the equations (1) have been obtained at time $=0.1$ for the above three cases, using the present schemes for different grid sizes. Fig. $2 \mathrm{~b}$ shows the computed values of $u$-velocity component for two different grids; $(10 \times 5)$, $(40 \times 20)$, using the compact ADI scheme. The fourth-order accurate compact ADI scheme is capable of producing convergent and stable steady-state solutions with severe gradient 
even on relatively coarse grid size and large time step size $(\Delta t=0.01)$, in comparison with the fourth-order Du Fort Frankel scheme that required finer grid $(80 \times 40)$, and smaller time step size $(\Delta t=0.0002)$, as shown in Fig. 3. Moreover, the the fourth-order Du Fort Frankel scheme exhibits overshoots at the steep gradients especially with a coarse grid. This indicates that the fourth-order Du Fort Frankel scheme is unstable, and a much smaller time step and grid step sizes than allowed by the linear stability condition are required. To test the effect of the initial conditions on the performance of the present schemes, initial conditions different from the ones given by equation (3.1), $u_{i j}=1$ \&

$v_{i j}=y / y_{M}$, are used. The results obtained by the compact ADI scheme are stable and convergent, while the fourth-order Du Fort Frankel scheme suffers from overshoots at the gradients, as shown in Fig. 3b. The computational efficiency of the two schemes has been tested by measuring the execution times, using PC-80486 DX2/66, necessary to obtain steady-state solutions at time $=0.1$, and by computing the two-dimensional error norms $\left(E_{u}, E_{v}\right)$ defined by:

$$
E_{u}=\frac{1}{N M} \sum_{i=1}^{N} \sum_{j=1}^{M}\left|u_{i j}-u_{s}\right|, \quad E_{v}=\frac{1}{N M} \sum_{i=1}^{N} \sum_{j=1}^{M}\left|v_{i j}-v_{s}\right|,
$$

where $\left(u_{i j}, v_{i j}\right)$ represent the numerical computed solutions and $\left(u_{s}, v_{s}\right)$ the exact solutions. Table 1 shows a comparison of the execution times and the error norms of the present schemes; the compact ADI scheme and the fourth-order Du Fort Frankel scheme. The compact ADI scheme computations with $\Delta t_{\max }=0.01$ required about 12.5 seconds, in comparison with 26 seconds needed by the fourth-order Du Fort Frankel scheme with $\Delta t_{\max }=0.0002$, for the same grid $(40 \times 20)$. Moreover, the compact ADI scheme produces more accurate and hence more efficient solution, even on a courase grid, as shown in Table 1. This concludes that the fourth-order accurate compact ADI scheme is twice more economical and more accurate than the fourth-order accurate Du Fort Frankel scheme.

\section{Problem case-2}

In this test case, we consider the solution of 2-D unsteady coupled Burgers' equations (1), that is dominated by internal gradients, in the domain $\{0<x<1,0<y<1, t>0\}$, with the following initial and Dirichlet boundary conditions [50]:

$$
\begin{aligned}
& u(x, y, 0)=\sin (\pi x) \sin (\pi y), \\
& v(x, y, 0)=\{\sin (\pi x)+\sin (2 \pi x)\}\{\sin (\pi y)+\sin (2 \pi y)\}, \\
& u(0, y, t)=u(1, y, t)=u(x, 0, t)=u(x, 1, t)=0, \\
& v(0, y, t)=v(1, y, t)=v(x, 0, t)=v(x, 1, t)=0 .
\end{aligned}
$$

The computed values of the velocity components $u$ and $v$, for the case of $R e=1$ and at different times $(t=0,0.01,0.05)$, on two different grids $(40 \times 40),(10 \times 10)$, using the compact ADI scheme, are shown in Fig. 4. Again, the fourth-order compact ADI scheme is capable of producing stable and accurate solution with internal gradient on a coarse grid $(10 \times 10)$, and with comparable accuracy to the solution on a refined grid 
$(40 \times 40)$. Tables $2-3$ show a comparison of the computed solutions using the present two schemes, with the previous numerical results of Arminjon and Beauchamp [50], using the finite element and the method of lines. The comparison shows that the solutions obtained by the compact ADI scheme, and by both the finite element and the method of lines coincide to three significant digits in most cases. The behaviour of the solution for $u$ and $\mathrm{v}$ components at larger time is also considered. The computed solution decreases very rapidly to zero at time $=0.5$, which is the same result obtained previously by Arminjon and Beauchamp [50]. Moreover, the present compact ADI scheme has the advantage over the other schemes that it can use a coarse grid and time step size at least twice as larger as for the other schemes to get convergent and accurate solution. Fig. 5 shows a comparison of the computed solutions for v-velocity at time $=0.01$, using the compact ADI scheme, and using the fourth-order Du Fort Frankel scheme that required finer grid $(40 \times 40)$ and smaller time step size $\left(\Delta t=10^{-6}\right)$ to produce stable solutions. Also, the execution times, using PC-80486 DX2/66, necessary to obtain stable solutions at time $=0.1$ and $R e=1$ for the present two schemes, are listed in Table 4. The compact ADI scheme computations, with $\Delta t_{\max }=10^{-3}$, required about 85 seconds, while the corresponding computations with the fourth-order Du Fort Frankel scheme, with $\Delta t_{\max }=10^{-6}$, required 780 seconds, for the same grid $(40 \times 40)$. This indicates that the present fourth-order accurate compact ADI scheme has higher computational efficiency for solving the unsteady coupled Burgers' equations with internal gradients.

\section{Conclusion}

In conclusion, the fourth-order accurate two-point compact ADI scheme and the fourthorder accurate Du Fort Frankel scheme are used to solve the two-dimensional unsteady coupled Burgers' equations, for problems that are dominated by moderate to severe internal and boundary gradients. The accuracy and the computational efficiency of the

present schemes are tested. The compact ADI scheme is found to be stable, efficient, and with better resolution of steep gradients in comparison with the other scheme, and with the previous numerical results of Arminjon and Beauchamp, using the finite element and the method of lines [50].

\section{Acknowledgments}

The author thank the reviewers for their valuable comments, which improved the quality of this paper. 
S.F. Radwan

Table 1. Comparison of execution times and error norms for computed solutions of 2-D coupled Burgers' equations at time $=0.1 \& R e=50$, for problem case- 1 .

\begin{tabular}{|c|c|c|c|c|c|c|}
\hline & \multicolumn{3}{|c|}{ 4-th compact scheme } & \multicolumn{3}{|c|}{ 4-th Du Fort Frankel scheme } \\
\hline $\begin{array}{l}\text { grind } \\
\text { points }\end{array}$ & $\begin{array}{l}\text { execution time } \\
\text { (seconds) }\end{array}$ & $-\log E_{u}$ & $-\log E_{v}$ & $\begin{array}{l}\text { execution time } \\
\text { (seconds) }\end{array}$ & $-\log E_{u}$ & $-\log E_{v}$ \\
\hline $10 \times 5$ & $\begin{array}{c}\Delta t=0.01 \\
\text { time }=1\end{array}$ & 12.0 & 11.4 & $\begin{array}{c}\Delta t=0.0002 \\
\text { time }=2.5\end{array}$ & 7.4 & 5.4 \\
\hline $20 \times 10$ & $\begin{array}{c}\Delta t=0.01 \\
\text { time }=3\end{array}$ & 13.6 & 13.3 & $\begin{array}{c}\Delta t=0.0002 \\
\text { time }=7\end{array}$ & 5.4 & 4.6 \\
\hline $40 \times 20$ & $\begin{array}{c}\Delta t=0.01 \\
\text { time }=12.5\end{array}$ & 13.8 & 13.9 & $\begin{array}{c}\Delta t=0.0002 \\
\text { time }=26\end{array}$ & 4.8 & 4.2 \\
\hline $80 \times 40$ & $\begin{array}{l}\Delta t=0.01 \\
\text { time }=42\end{array}$ & 14.8 & 15.4 & $\begin{array}{c}\Delta t=0.0002 \\
\text { time }=100\end{array}$ & 4.5 & 4.1 \\
\hline
\end{tabular}

Table 2. Comparison of the values of $u$ and $v$ computed by the Compact ADI scheme and Du Fort Frankel scheme at time $=0.01 \& R e=1$, for problem case- 2 .

\begin{tabular}{|c|c|c|c|c|c|c|}
\hline points & \multicolumn{3}{|c|}{ compact ADI scheme } & \multicolumn{3}{c|}{4 -th Du Fort Frankel scheme } \\
\hline & $N=10$ & $N=20$ & $N=40$ & $N=10$ & $N=20$ & $N=40$ \\
& $\Delta t=1 / 800$ & $1 / 800$ & $1 / 1000$ & $\Delta t=10^{-6}$ & $10^{-6}$ & $10^{-6}$ \\
\hline & \multicolumn{3}{|c|}{ the velocity $u$} & \multicolumn{3}{c|}{ the velocity u } \\
$(0.1,0.1)$ & 0.07320 & 0.07275 & 0.07273 & 0.09549 & 0.08446 & 0.07729 \\
$(0.2,0.8)$ & 0.27800 & 0.27803 & 0.27800 & 0.30231 & 0.28314 & 0.27929 \\
$(0.4,0.4)$ & 0.72292 & 0.72290 & 0.72285 & 0.72942 & 0.72215 & 0.72183 \\
$(0.7,0.1)$ & 0.20542 & 0.20506 & 0.20497 & 0.25000 & 0.21957 & 0.20978 \\
$(0.9,0.9)$ & 0.07968 & 0.07955 & 0.07953 & 0.09549 & 0.08785 & 0.08276 \\
\hline & \multicolumn{3}{|c}{ the velocity $v$} & & \multicolumn{3}{c|}{ the velocity $v$} \\
$(0.1,0.1)$ & 0.43599 & 0.43662 & 0.43448 & 0.80425 & 0.63005 & 0.51128 \\
$(0.2,0.8)$ & -0.13444 & -0.13131 & -0.13148 & -0.29180 & -0.16784 & -0.13866 \\
$(0.4,0.4)$ & 1.65503 & 1.65869 & 1.65917 & 1.66391 & 1.65563 & 1.65177 \\
$(0.7,0.1)$ & 0.06486 & 0.06337 & 0.06417 & -0.12738 & 0.00655 & 0.04585 \\
$(0.9,0.9)$ & 0.01427 & 0.01512 & 0.01476 & 0.07771 & 0.04794 & 0.02743 \\
\hline
\end{tabular}


Table 3. Comparison of the values of $u$ and $v$ computed by the method of lines and the finite element method [50] at time $=0.01 \& R e=1$, for problem case- 2 .

\begin{tabular}{|c|c|c|c|c|c|c|}
\hline points & \multicolumn{3}{|c|}{ method of lines } & \multicolumn{3}{c|}{ finite element method } \\
\hline & $N=10$ & $N=20$ & $N=40$ & $N=10$ & $N=20$ & $N=40$ \\
& $\Delta t=1 / 800$ & $1 / 2000$ & $1 / 4000$ & $\Delta t=1 / 2000$ & $1 / 1500$ & $1 / 2000$ \\
\hline & \multicolumn{3}{|c|}{ the velocity $u$} & \multicolumn{3}{c|}{ the velocity $u$} \\
$(0.1,0.1)$ & 0.07277 & 0.07257 & 0.07253 & 0.07279 & 0.07257 & 0.07252 \\
$(0.2,0.8)$ & 0.28887 & 0.28846 & 0.28836 & 0.28867 & 0.28842 & 0.28835 \\
$(0.4,0.4)$ & 0.72315 & 0.72205 & 0.72178 & 0.72370 & 0.72210 & 0.72179 \\
$(0.7,0.1)$ & 0.20139 & 0.20112 & 0.20106 & 0.20157 & 0.20117 & 0.20107 \\
$(0.9,0.9)$ & 0.07956 & 0.07948 & 0.07947 & 0.07951 & 0.07947 & 0.07946 \\
\hline & \multicolumn{3}{|c|}{ the velocity $v$} & \multicolumn{3}{c|}{ the velocity $v$} \\
$(0.1,0.1)$ & 0.43857 & 0.43302 & 0.43173 & 0.44130 & 0.443357 & 0.43178 \\
$(0.2,0.8)$ & -0.13200 & -0.12387 & -0.12184 & -0.13172 & -0.12366 & -0.12180 \\
$(0.4,0.4)$ & 1.66509 & 1.65571 & 1.65335 & 1.66212 & 1.65499 & 1.65316 \\
$(0.7,0.1)$ & 0.06137 & 0.06571 & 0.06679 & 0.06306 & 0.06621 & 0.06692 \\
$(0.9,0.9)$ & 0.01459 & 0.01372 & 0.01349 & 0.01459 & 0.01367 & 0.01349 \\
\hline
\end{tabular}

Table 4. Comparison of execution times for computed solutions of 2-D coupled Burgers' equations at time $=0.01 \& R e=1.0$ for problem case- 2 .

\begin{tabular}{|c|c|c|}
\hline grid points & 4-th compact ADI scheme & 4-th Du Fort Frankel Scheme \\
\hline $10 \times 10$ & $\begin{array}{c}\Delta t=1.25 \times 10^{-3} \\
\text { time }=4 \mathrm{sec}\end{array}$ & $\begin{array}{c}\Delta t=10^{-6} \\
\text { time }=35 \mathrm{sec}\end{array}$ \\
\hline $20 \times 20$ & $\begin{array}{c}\Delta t=1.25 \times 10^{-3} \\
\text { time }=14 \mathrm{sec}\end{array}$ & $\begin{array}{c}\Delta t=10^{-6} \\
\text { time }=240 \mathrm{sec}\end{array}$ \\
\hline $40 \times 40$ & $\begin{array}{c}\Delta t=10^{-3} \\
\text { time }=85 \mathrm{sec}\end{array}$ & $\begin{array}{c}\Delta t=10^{-6} \\
\text { time }=780 \mathrm{sec}\end{array}$ \\
\hline
\end{tabular}

\section{References}

[1] Fanchi J.R., Multidimensional Numerical Dispersion, SPE J., 1983, V.23, 143.

[2] Peaceman D.W., Fundamentals of Numerical Reservior Simulations, Elsevier Science Publishers, Amsterdam, 1977.

[3] Celia M.A. and Gray W.G., Numerical Methods for Differntial Equations, Prentice Hall, Englewood Cliffs, NJ, 1992.

[4] Sharif M.A.R. and Busnaina A.A., Assessment of Finite Difference Approximations for the Advection Terms in the Simulation of Practical Flow Problems, J. Comput. Phys., 1988 , V.74, 143. 
[5] Leonard B.P., Order of Accuracy of Quick and Related Convection-Diffusion Schemes, Appl. Math. Modeling 1995, V.19, 640.

[6] Liu J., Pope G.A. and Sepehrnoori K., A High-Resolution Finite-Difference Scheme for Nonuniform Grids, Appl. Math. Modeling, 1995, V.19, 162.

[7] Radwan S.F., On the Higher-Order Accurate Scheme for Solving Two-Dimensional Unsteady Burgers' Equation, in Proceeding of the International Congress on Fluid Dynamics and Propulsion, Cairo Univ., Egypt, 1996, Vol.III, 788.

[8] Harten A., On a Class of High Resolution Total Variation Stable Finite Difference Schemes, J. Numer. Anal., 1984, V.21, 18.

[9] Yee H.C., Construction of Explicit and Implicit Symmetric TVD Schemes and Their Applications, J. Comput. Phys., 1987, V.68, 151.

[10] Datta Gupta A., Lake L.W., Pope G.A. and Sepehrnoori K., High Resolution Monotonic Schemes for Reservoir Fluid Flow Simulation, In. Situ, 1991, V.15, 235.

[11] Orszag S.A. and Israeli M., Numerical Simulation of Viscous Incompressible Flows, Annual Rev. of Fluid Mech., 1974, V.6, 281.

[12] Hirsh R.S., Higher-Order Accurate Difference Solutions of Fluid Mechanics Problems by a Compact Differencing Techinque, J. Comput. Phys., 1975, V.19, 90.

[13] Krause E., Hirschel E.H. and Kordulla W., Fourth-Order Mehrstellen Integration for ThreeDimensional Turbulent Boundary Layers, in AIAA Computational Fluid Dynamics Conference, July 1973, 1973, 92.

[14] Weinberg B.C., Leventhal S.H. and Ciment M., The Operator Compact Implicit Scheme for Viscous Flow Problems, AIAA paper No. 77-638, 1977.

[15] Ciment M., Leventhal S.H. and Weinberg B.C., The Operator Compact Implicit Method for Parabolic Equations, J. Comput. Phys., 1978, V.28, 135.

[16] Berger A.E., Solomon J.M., Ciment M., Leventhal S.H. and Weinberg B.C., Generalized OCI Schemes for Boundary Layer Problems, Math. of Computation, 1980, V.35(151), 695.

[17] Peters N., Boundary Layer Calculation by a Hermitian Finite Difference Method, in Proceedings of the Fourth International Conference on Numerical Methods in Fluid Mechanics, Volume 35 of Lecture Notes in Physics, Robert D. Richtmyer, edt., Springer-Verlag, 1975, 313.

[18] Rubin G. and Graves R.A., Viscous Flow Solutions with a Cubic Spline Approximation, Comput. \& Fluids, 1975, V.3, N 1, 1-36.

[19] Rubin S.G. and Graves R.A., A Cubic Spline Approximation for Problems in Fluid Mechanics, NASA TR R-436, 1975.

[20] Rubin S.G. and Khosla P.K., Higher-Order Numerical Solutions Using Cubic Splines, NASA CR2653, 1976 .

[21] Rubin S.G. and Khosla P.K., Higher-Order Numerical Methods Derived from Three-Point Polynomial Interpolation, NASA CR-2735, 1976.

[22] Adam Y., A Hermitian Finite Difference Method for the Solution of Parabolic Equations, Comput. \& Math. Appl., 1975, V.1, 393.

[23] Adam Y., Highly Accurate Compact Implicit Methods and Boundary Conditions, J. Comput. Phys., 1977, V.24, 10. 
[24] Gupta M.M., Manohar R.P. and Stephenson J.W., A Single Cell High Order Scheme for the Convection-Diffusion Equation with Variable Coefficients, Int. J. Numer. Methods Fluids, 1984, V.4, 641.

[25] Gupta M.M., High Accuracy Solutions of Incompressible Navier-Stokes Equations, J. Comput. Phys., 1991, V.93, 343.

[26] Dennis S.C.R. and Hudson J.D., Compact $h^{4}$-Finite-Difference Approximations to Operators of Navier-Stokes Type, J. Comput. Phys., 1989, V.85, 390.

[27] Li M., Tang T. and Fornberg B., A Compact Fourth-Order Finite Difference Scheme for the Steady Incompressible Navier-Stokes Equations, Int. J. Numer. Methods Fluids, 1995, V.20, 10.

[28] Spotz W.F. and Carey G.F., High-Order Compact Scheme for the Steady Stream Function Vorticity Equations, Int. J. Numer. Methods Eng., 1995, V.38, 3497.

[29] Johnson R.W. and McKinnon R.J., Differential Equation Based Representation of Truncation Errors for Accurate Numerical Simulation, Int. J. Numer. Methods Fluids, 1991, V.13, 739.

[30] Shu C.W., Nonlinearly Stable Compact Schemes for Shock Calculations, SIAM. J. Num. Anal., 1994, V.31, 607 .

[31] Yu S.T., On the Accuracy of Compact Differencing Schemes for DNS, NASA Conference Publication, Proceeding of the Workshop on Computational Turbulence Modeling, Sep. 15-16, 1993, Cleveland, $\mathrm{OH}$, USA.

[32] Chen G. and Yang Z., Compact Second-Order Upwind Finite Difference Schemes for the ConvectionDiffusion Equations, J. Hydrodynamics, 1992, V.4, N 4, 32.

[33] Gottlieb D. and Abarbanel S., Stable and Accurate Boundary Treatments for Compact High-Order Finite Difference Schemes, Appl. Numer. Math., 1993, V.12, N 1-3, 55.

[34] Zhang J., Multigrid Solution of the Convection-Diffusion Equation with High Reynolds Number, in Prel. Proceeding Copper Mountain Conference on Iterative Methods, April 9-13, 1996.

[35] Zhang J., On Convergence and Performance of Iterative Methods with Fourth-Order Compact Schemes, Numer. Methods for Partial Diff. Eqns., 1998, V.14, N 2, 263.

[36] Yavneh I., Analysis of a Fourth-Order Compact Scheme for Convection-Diffusion, J. Comput. Phys., 1997, V.133, 361.

[37] Weinan E. and Liu J., Essentially Compact Schemes for Unsteady Viscous Incompressible Flows, J. Comput. Phys., 1996, V.126, 122.

[38] Lele S.K., Compact Finite Difference Schemes with Spectral-Like Resolution, J. Comput. Phys., 1992, V.103, 16.

[39] White A.B., Numerical Solution of Two-Point Boundary-Value Problems, Ph. D. Thesis, California Inst. of Technology, USA, 1974.

[40] Keller H.B., Numerical Solution of Boundary Value Problems for Ordinary Differential Equations; Survey and Some Recent Results on Difference Methods, A.K. Aziz, ed., Academic press, Inc., 1975. 27.

[41] Liniger W. and Willoughby R.A., Efficint Integration Methods for Stiff Systems of Ordinary Differential Equations, SIAM. J. Num. Anal., 1970, V.7, 47.

[42] Radwan S.F., On the Second Diagonal Padé Approximation for Solving Fluid Dynamics Problems, in Proceeding of Assiut 1-st International Conf. of Math. and Statistics, Assiut Univ., Egypt, Part VII, 1990, 65 . 
[43] Radwan S.F., Fourth-Order Accurate Calculations of the Three-Dimensional Compressible Boundary Layers on Aerospace Configurations, AIAA Paper No. 88-2522-CP, 1988.

[44] Wornom S.F., Critical Study of Higher-Order Numerical Methods for Solving the Boundary-Layer Equations, NASA CR Paper 1302, also, AIAA Paper No. 77-637, 1978.

[45] Whitham G.B., Linear and Nonlinear Waves, John Wiley and Sons, New York, 1974.

[46] Rizun V.I. and Engel'brekht Iu.K., Application of the Burgers' Equation with a Variable Coefficient to Study of Nonplaner Wave Transients, PMM. J. Appl. Math., 1976, V.39, 524.

[47] Fried J.J. and Combarnous M.A., Dispersion in Porous Media, Adv. Hydrosci., 1971, V.7(169).

[48] Fletcher C.J., A Comparison of Finite Element and Finite Difference Solutions of the One- and Two-Dimensional Burgers' Equations, J. Comput. Phys., 1983, V.51, 159.

[49] M J.H., Ten Thije Boonkkamp and Verwer J.G., On the Odd-Even Hopscotch Scheme for the Numerical Integration of Time Dependent Partial Differential Equation, Appl. Numer. Math., 1987, V.3, 183.

[50] Arminjon P. and Beauchamp C., Numerical Solution of Burgers' Equations in Two-Space Dimensions, Computer Methods in Appl. Mech. and Eng., 1979, V.19, N 3, 351.

[51] Jain P.C. and Raja M., Splitting-Up Technique for Burgers' Equations, Indian J. Pure and Appl. Math., 1979, V.10, 1545.

[52] Jain P.C. and Lohar B.L., Cubic Spline Technique for Coupled Non-Linear Parabolic Equations, Comp. and Maths. with Appls., 1979, V.5, 179.

[53] El-Zoheiry H. and El-Naggar B.B., Numerical Study of the Two-Dimensional Burgers' Equations Using the Cubic Spline Approximations, in Proceeding of 2nd International Conf. on Eng. Math. and Physics, Cairo Univ., 1994, Vol.3, 363.

[54] EL-Naggar B.B., SADI Spline Alternating Direction Implicit Method for Solving the Coupled Burgers' Equations, Alexandria Eng. J., 1997, V.36(2), Section D, 19.

[55] Dahlquist G.G., A Special Stability Criterion for Linear Multistep Methods, BIT, Vol.3, 1963.

[56] Richtmyer R.D. and Morton K.W., Difference Methods for Initial-Value Problems, 2nd Edition, Wiley Interscience, NY, 1967.

[57] Kreiss G. and Kreiss H., Convergence to Steady State of Solution of Burgers' Equation, ICASE Report No. 85-50, NASA LRC, Hampton, Virginia, USA, 1985. 


\section{Figure Captions}

Figure 1: The couputed amplification factors (2.22) of the numerical solution of coupled Burgers' equations (1), using the explicit fourth-order accurate Du Fort Frankel scheme for different values of $|c|$ and $d$. 
Figure 2a: The excact steady state solutions for $u$ and $v$ velocities of the coupled unsteady Burgers' equations for problem case-1. 
Figure 2b: The computed steady state solutions for $u$-velocity of the coupled Burgers' equations for problem case-1, for two different grid sizes; $(40 \times 20),(10 \times 5)$, using the present compact ADI scheme. 
Figure 3: The computed steady state solutions for $u$ and $v$ velocities of the coupled unsteady Burgers' equations for problem case-1b, with internal severe gradient, using compact ADI scheme, and Du Fort Frankel scheme. 
Figure 4: The computed solutions for $u$ and $v$ velocities of the coupled unsteady Burgers' equations for problem case- 2 at $t=0,0.01,0.05, \& R e=1$, for two different grid sizes $(40 \times 40),(10 \times 10)$, using the present compact ADI scheme. 
Figure 5: Comparison of the computed solutions of $v$-velocity component of the coupled unsteady Burgers' equations for problem case-2, at time $=0.01 \& R e=1$, using the present compact ADI scheme, and Du Fort Frankel scheme. 


$$
\begin{array}{llll}
- & c=1.0 & \& & d=0.5 \\
\hdashline-- & c=0.5 & \& & d=0.5 \\
+++++ & c=.25 & \& c & d=0.5 \\
c=.25 & \& c & d=.01
\end{array}
$$

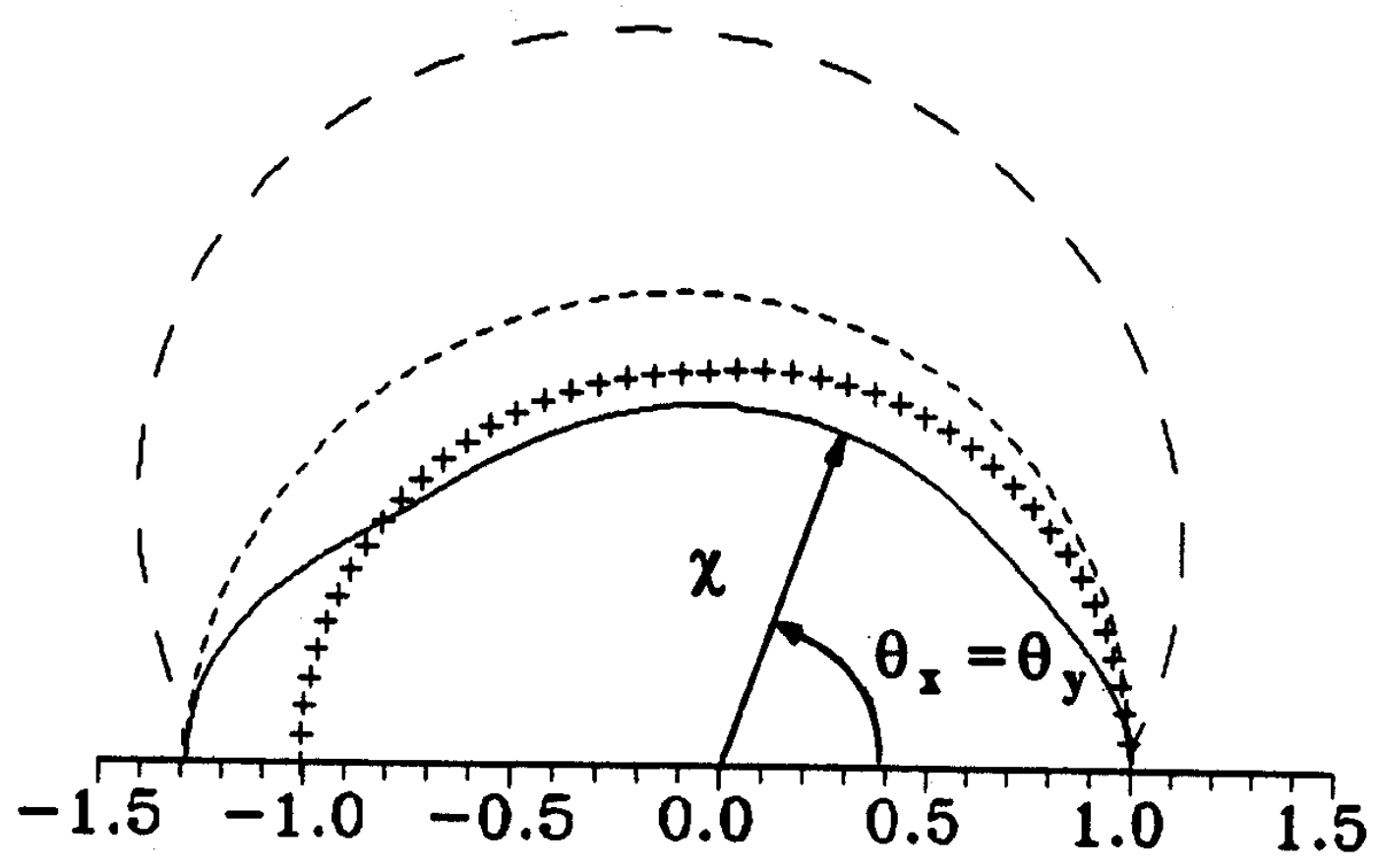

$$
\begin{array}{rlrl}
c=0.35 & \& & d & =0.5 \\
--- & \theta_{y} & =0 \\
& =\pi_{\pi} / 2
\end{array}
$$

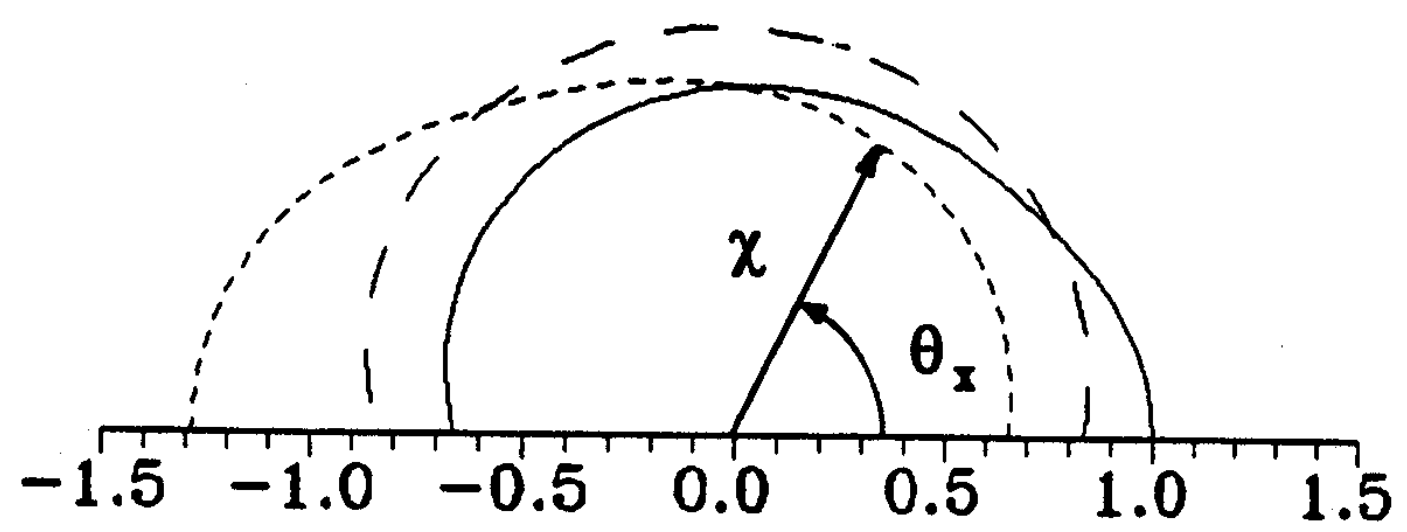



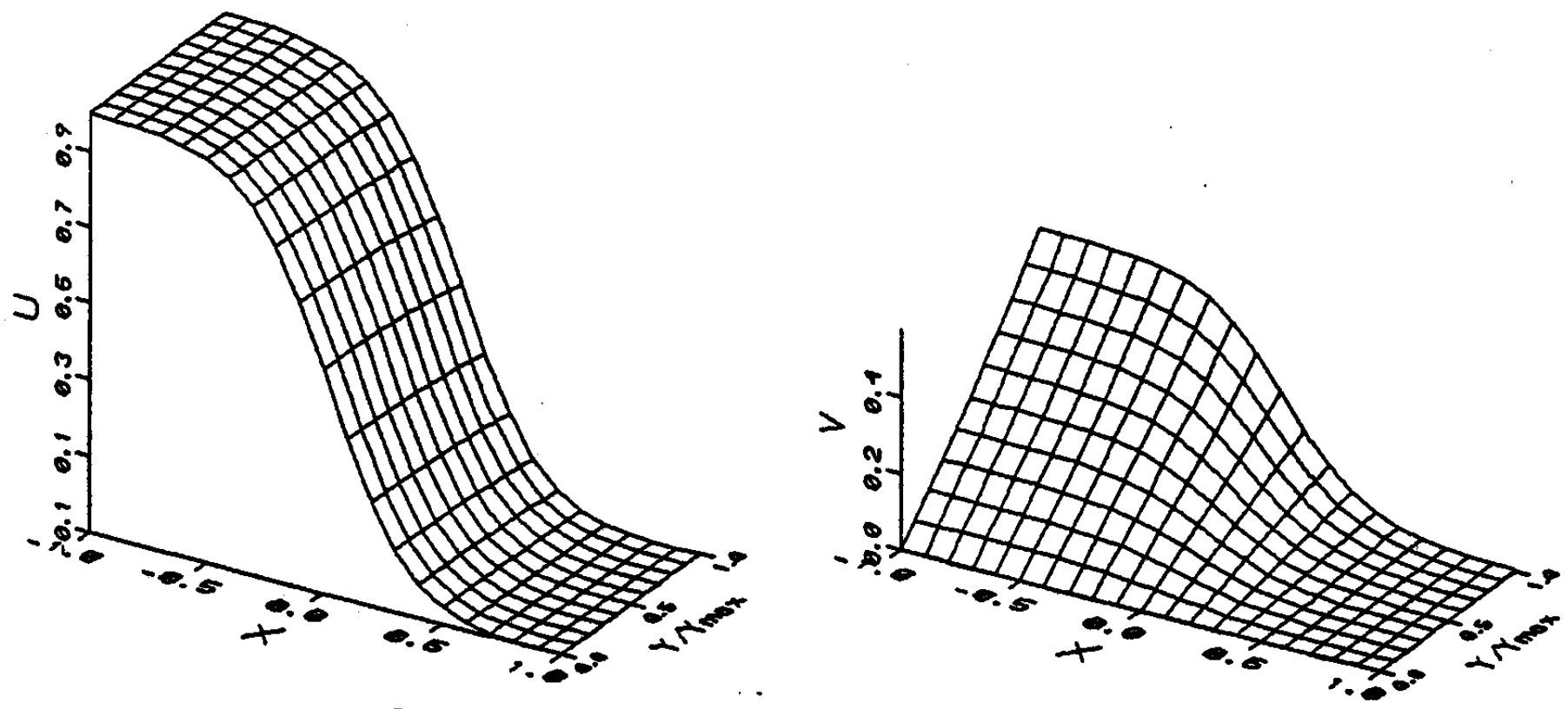

case 1a. . $\quad$ Re $=10 ., k=5, a_{0}=110.13$
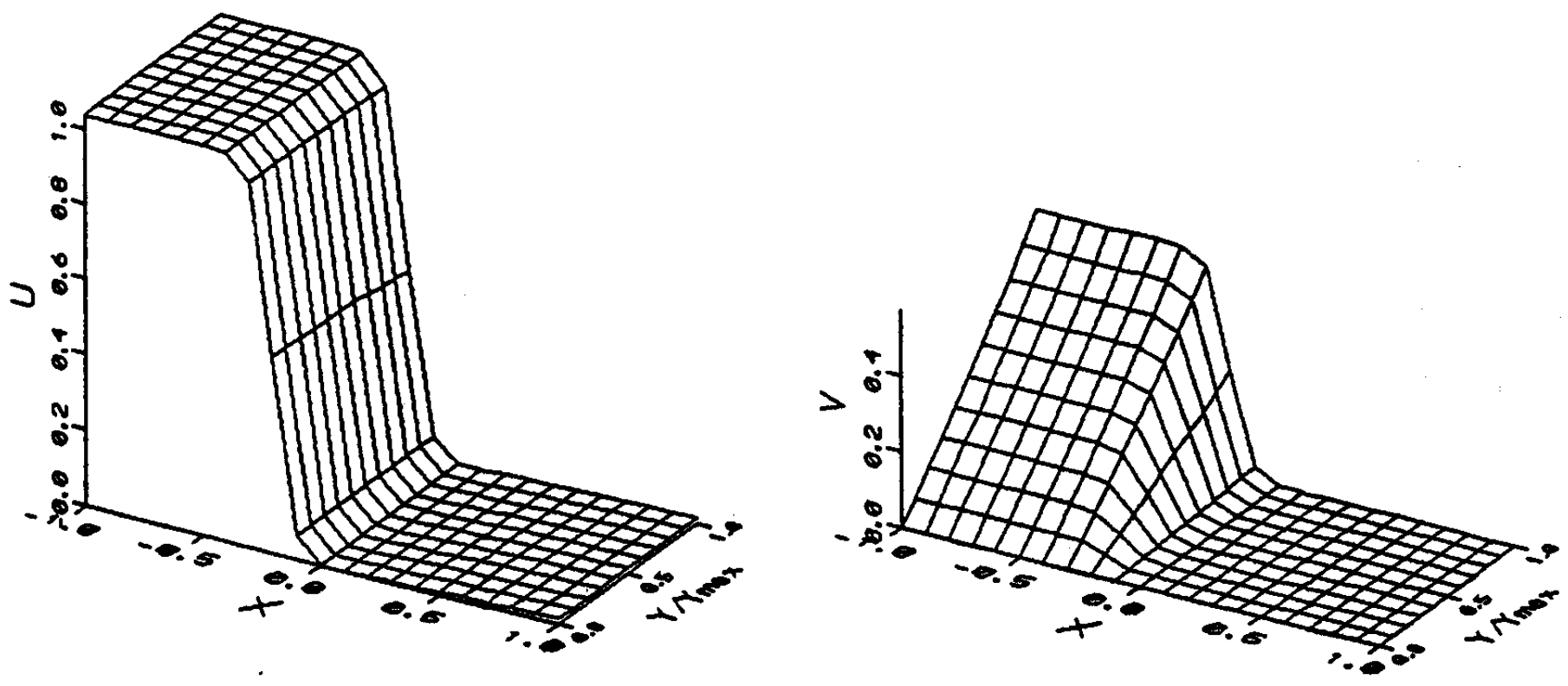

case 1b. $\quad \operatorname{Re}=50 ., k=25, a_{0}=1.29 \times 10^{13}$
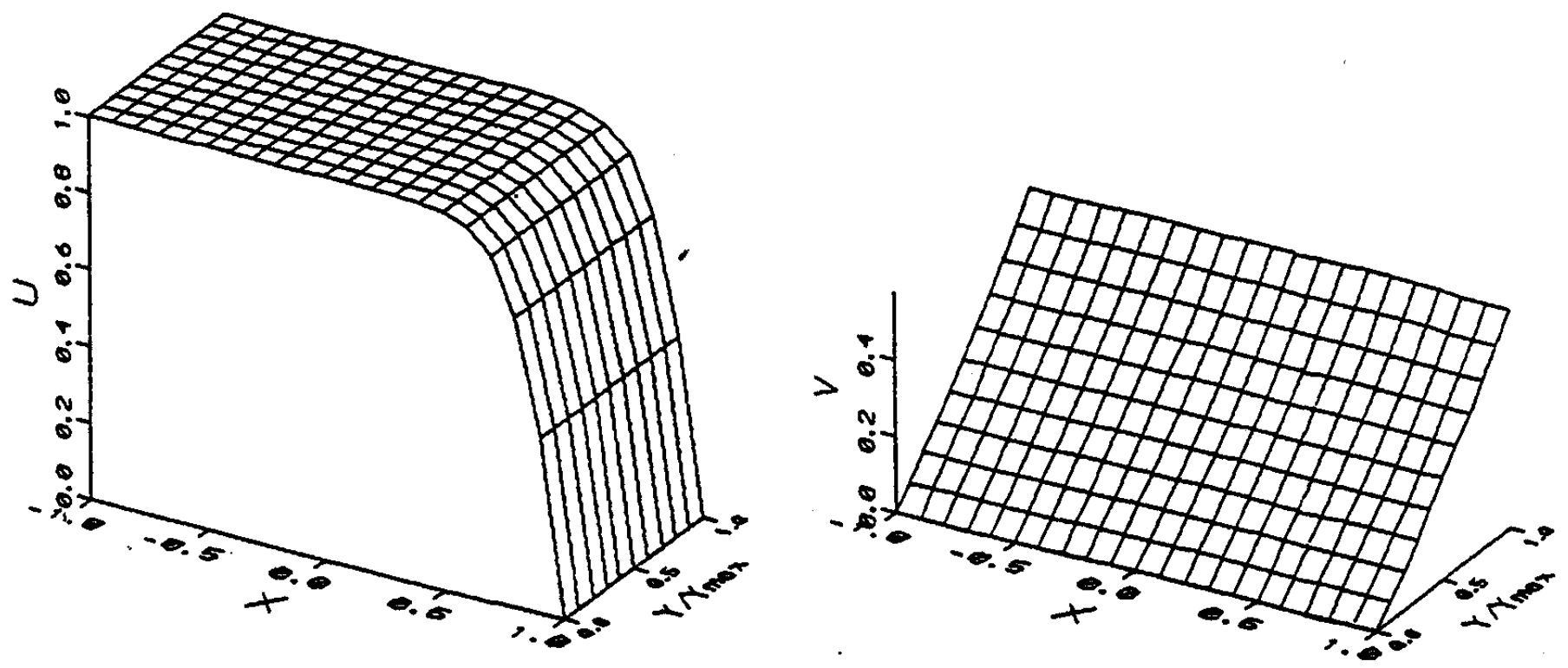

case 1c.

$R e=10, \quad k=5, a_{0}=.011013$ 

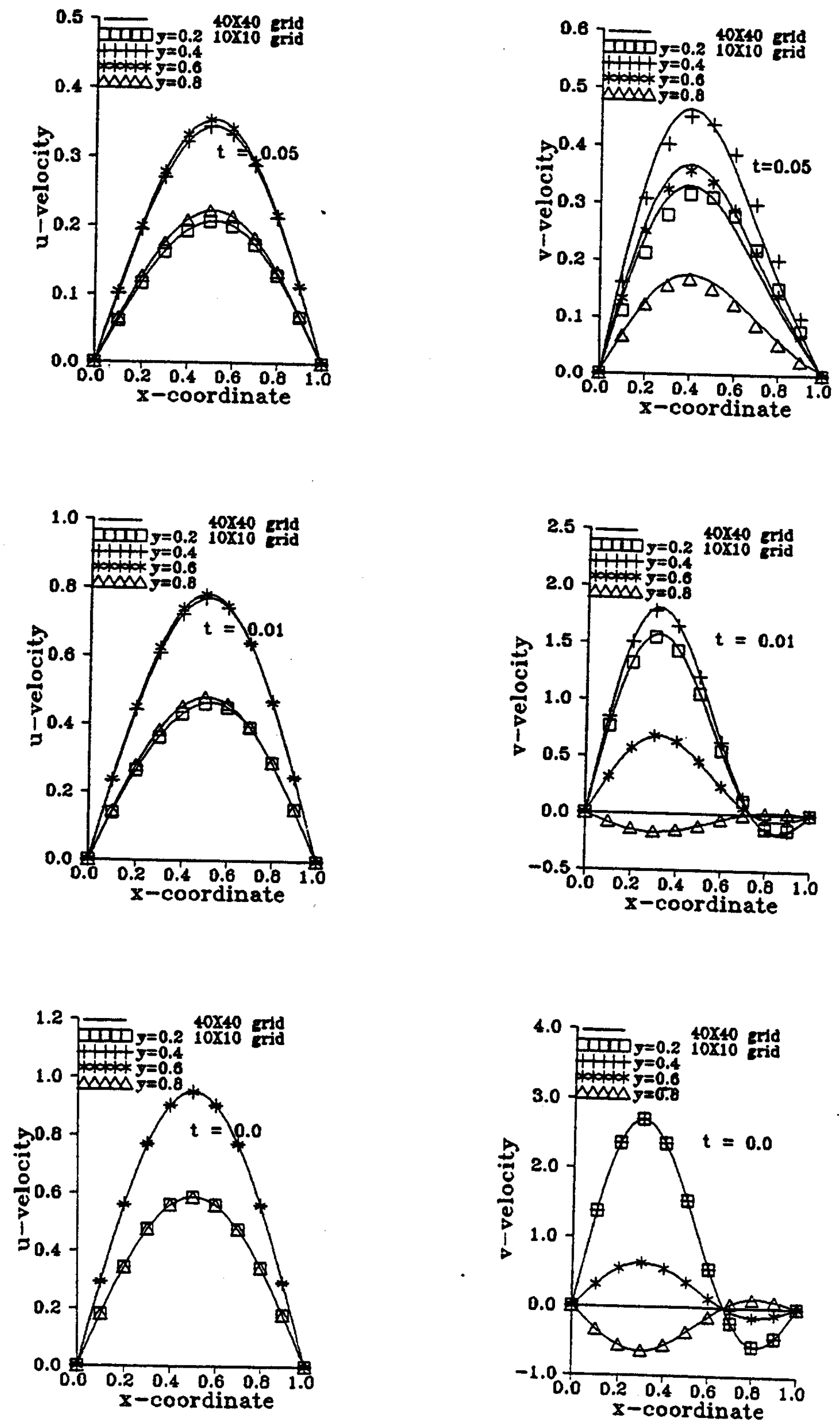

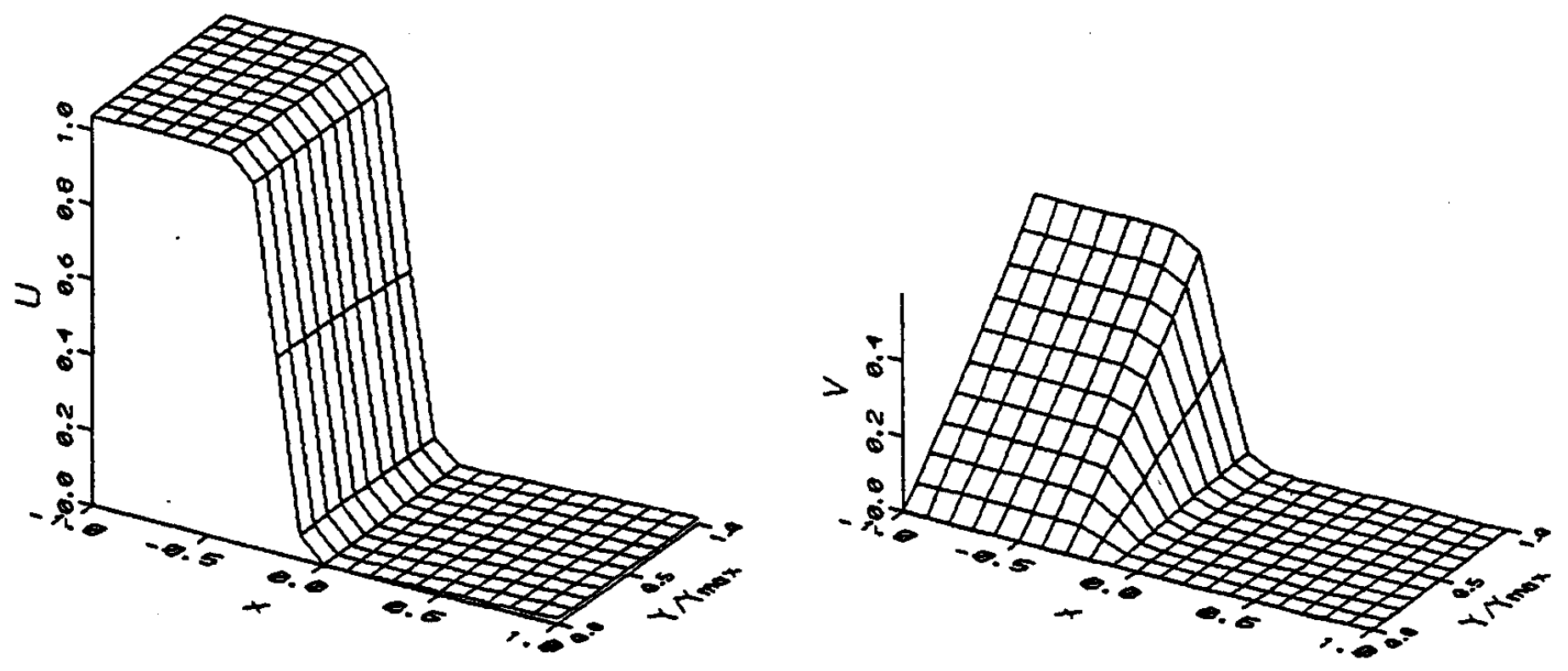

a. compact $A D I$ scheme (20x10).
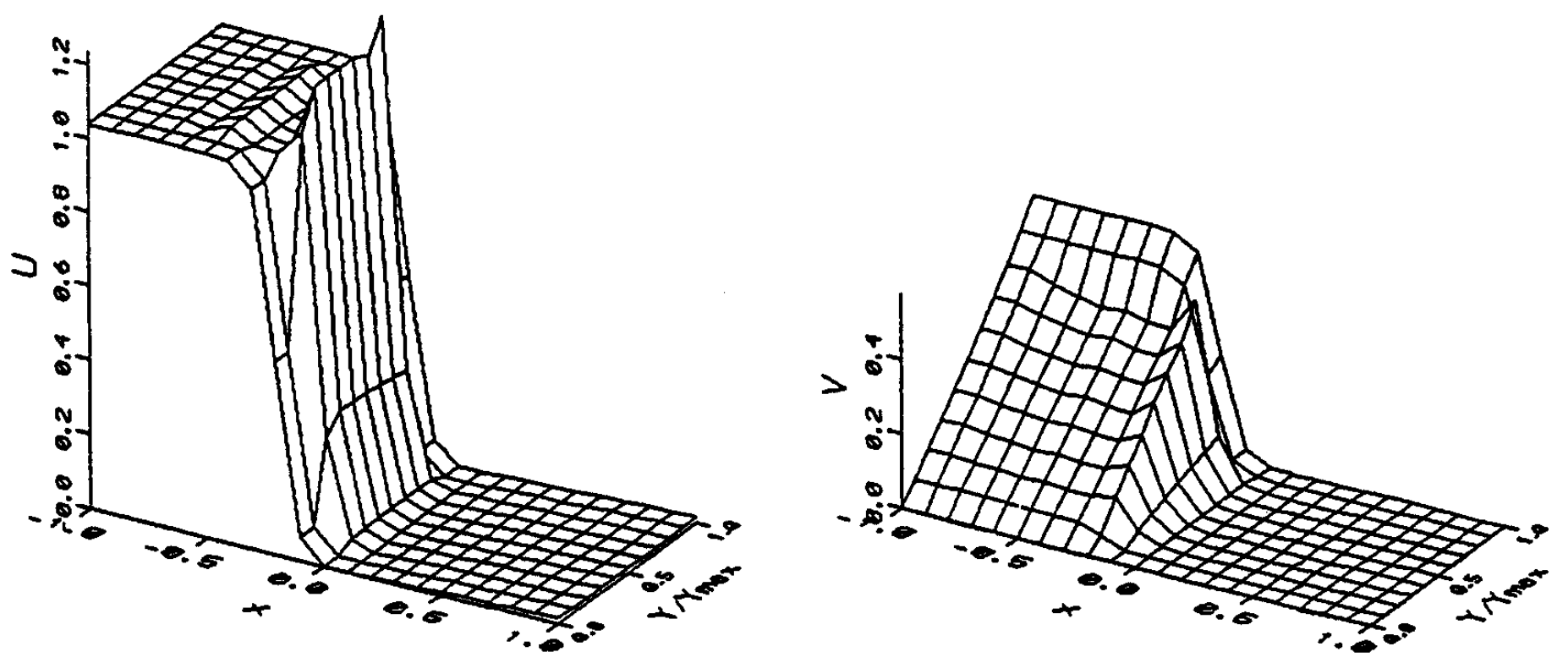

b. Du Fort Frankel scheme ( $20 \times 10)$.
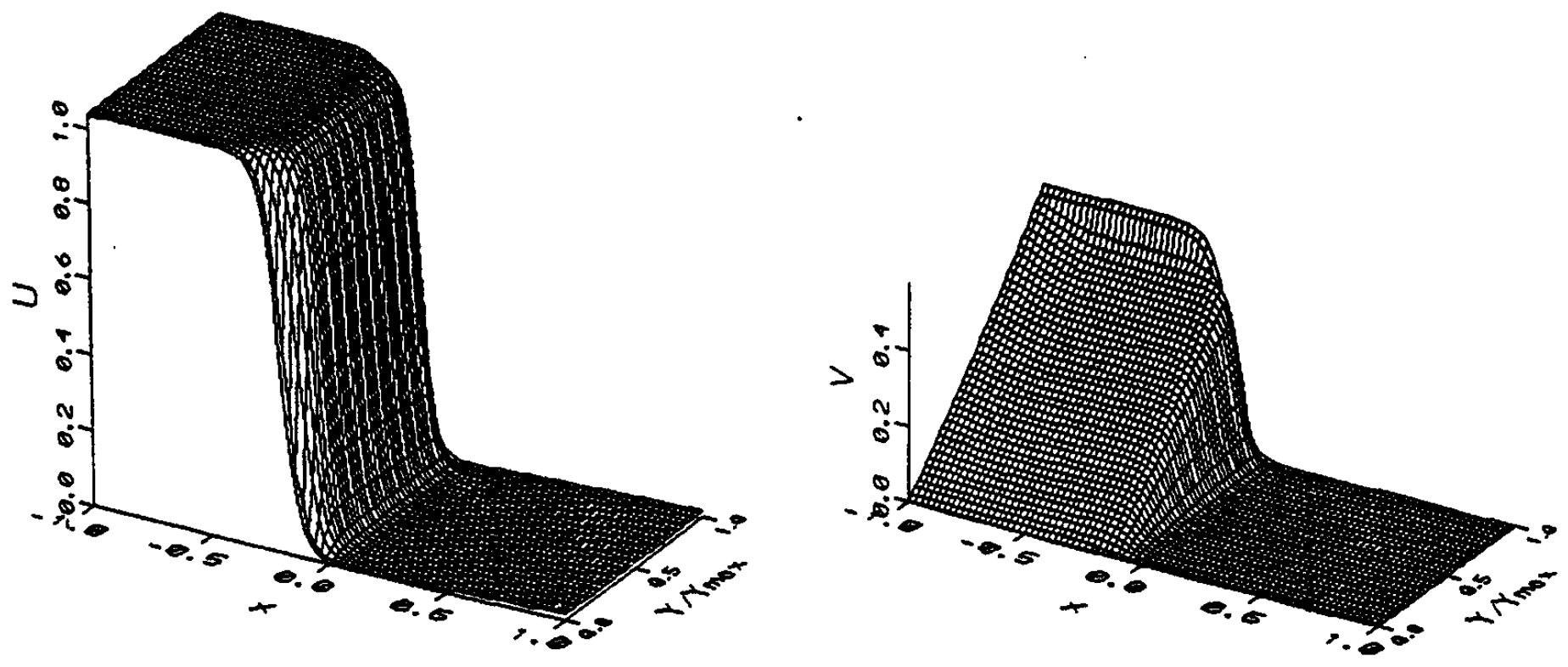

Du Fort Frankel scheme ( $80 \times 40)$. 

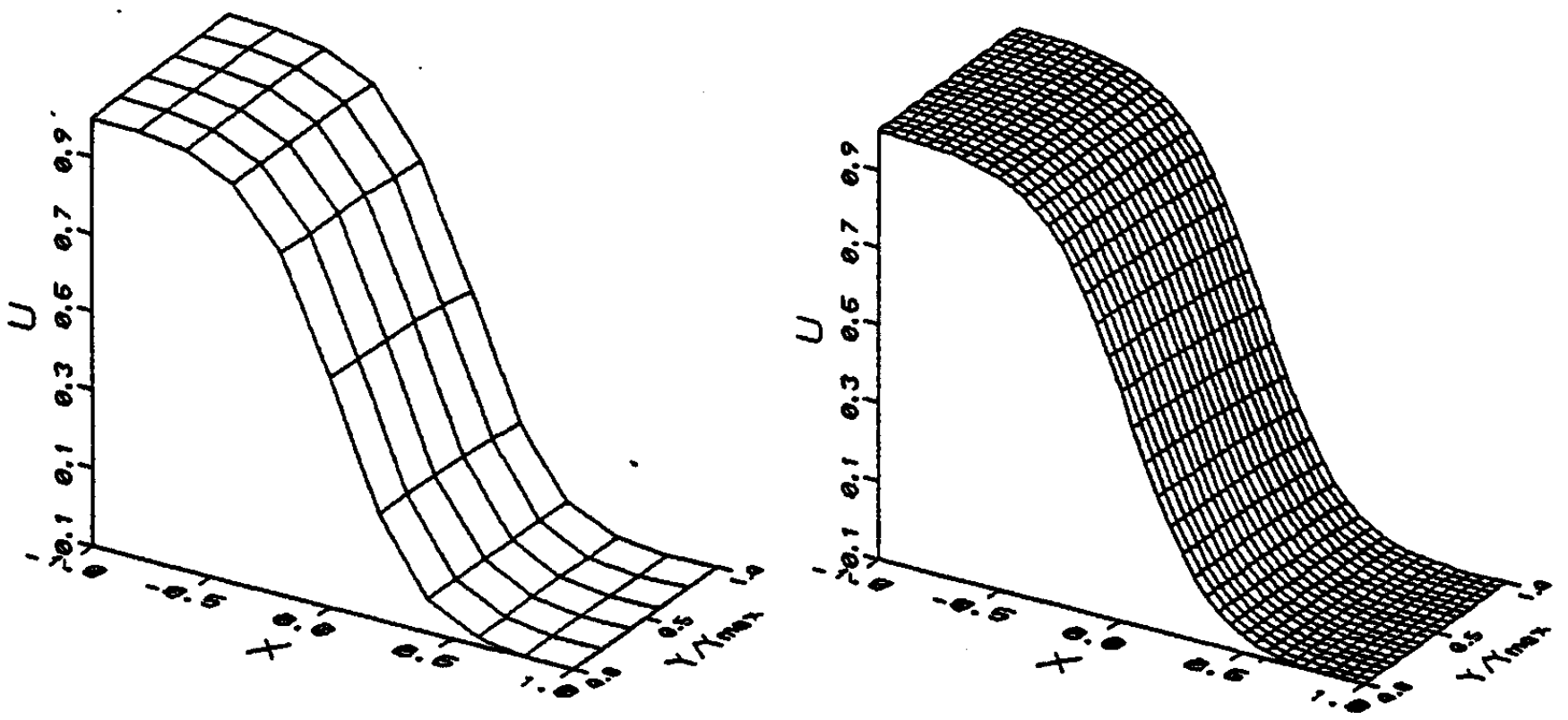

case $1 a$

$\operatorname{Re}=10, \quad k=5, a_{0}=110.13$
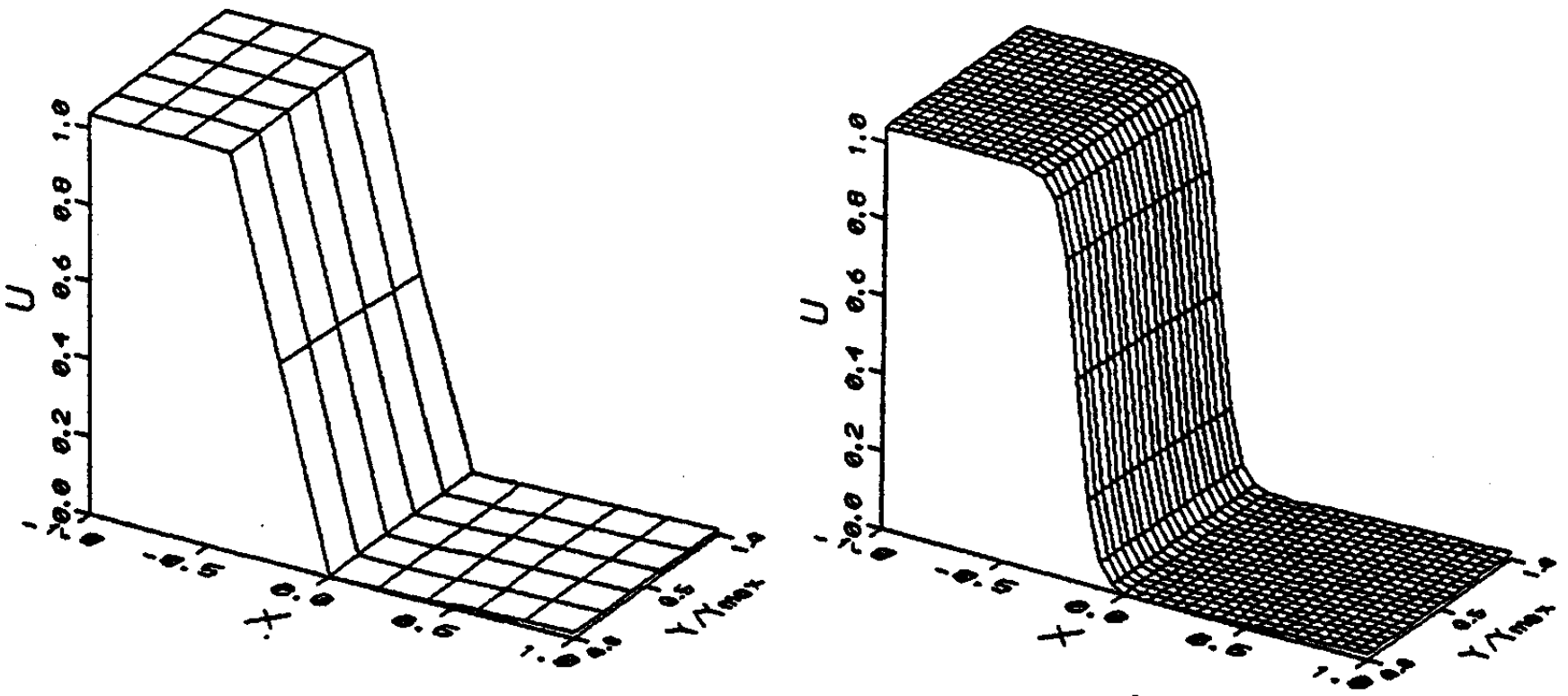

case $1 b$.

$\operatorname{Re}=50 ., k=25, a_{0}=1.29 \times 10^{13}$
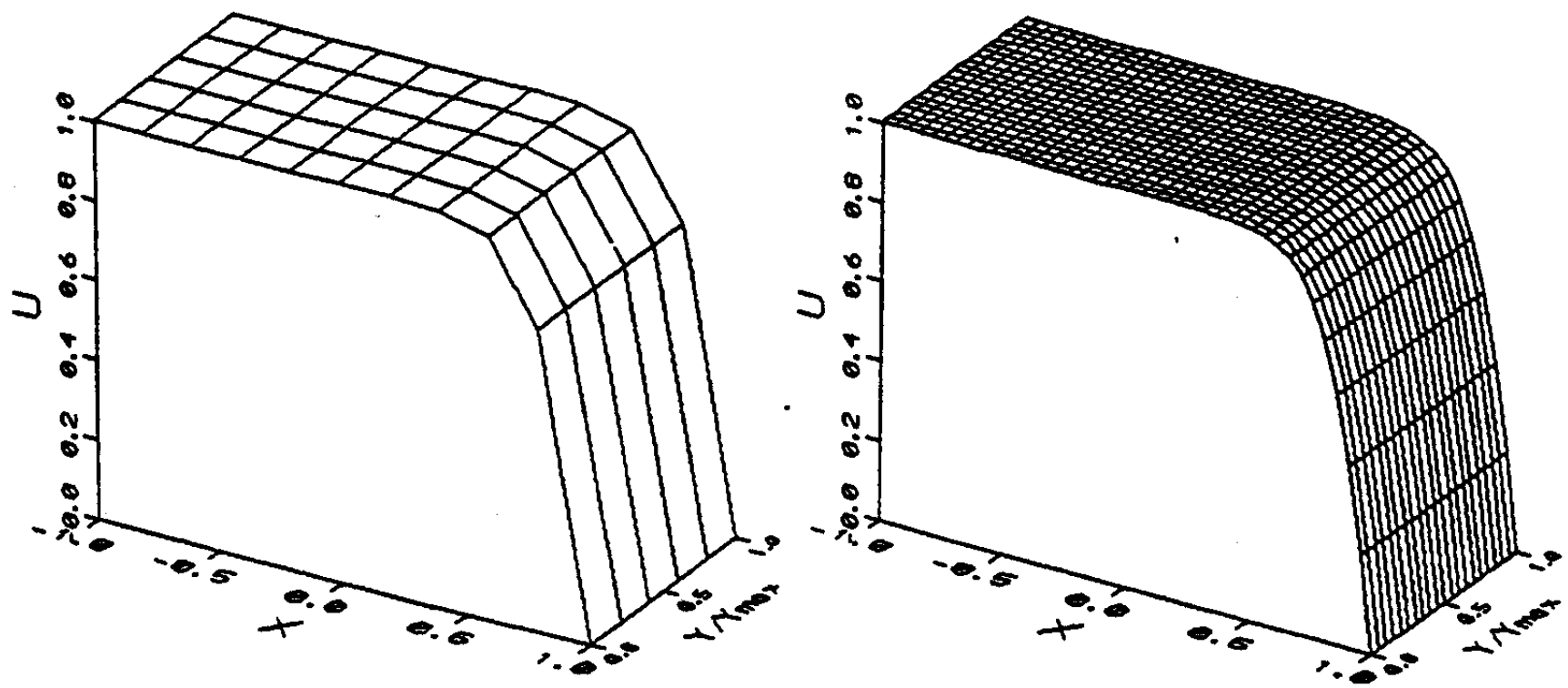

case 1c.

$\operatorname{Re}=10, \quad k=5, a_{0}=.011013$ 

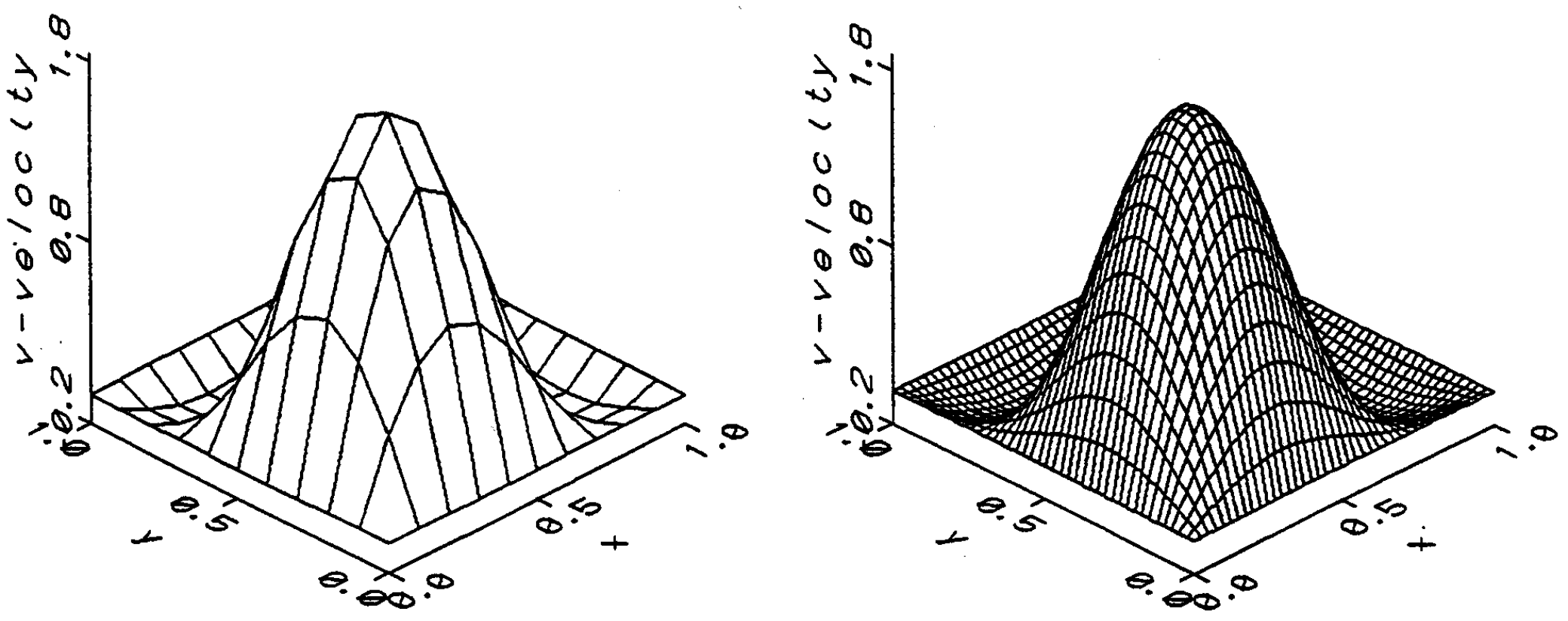

a. compact ADI scheme ( $10 \times 10$ \& $40 \times 40)$.
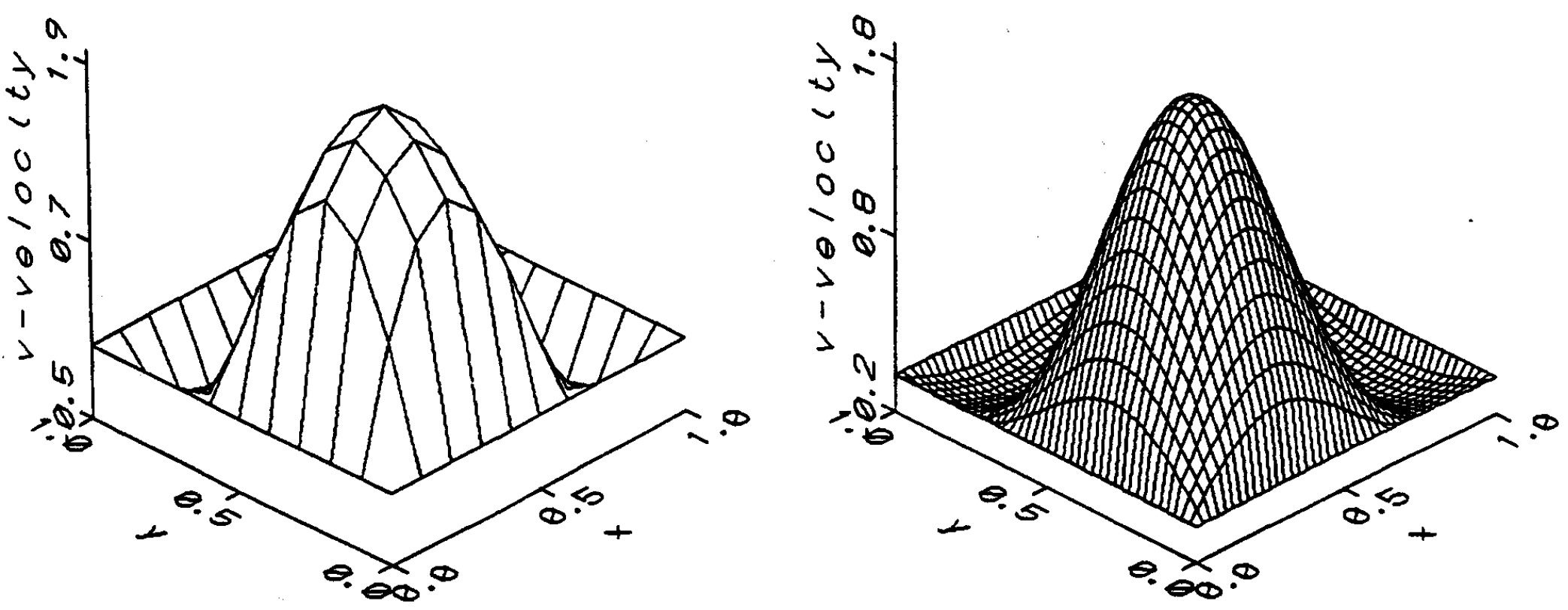

b. Du Fort Frankel scheme $(10 \times 10 \& 40 \times 40)$. 